Obere Extremität 2021 · 16:51-53 https://doi.org/10.1007/s11678-021-00625-0 Received: 19 September 2020

Accepted: 3 December 2020

Published online: 22 January 2021

(c) The Author(s) 2021



\section{Video online}

The online version of this article (https:// doi.org/10.1007/s11678-021-00625-0) contains the video: "Arthroscopic Knotless All-Suture Anchor Bankart Repair for Anterior Instability." You will find the video at the end of the article as "Supplementary material." Video by courtesy of A.-K. Tross, P.-C. Nolte, J.J. Ruzbarsky, B.P. Elrick, A.M. Johannsen, and P.J. Millett, The Steadman Clinic and Steadman Philippon Research Institute, Vail, CO, USA; all rights reserved 2020.

\section{Background}

Knotless, all-suture anchors are a valuable alternative to solid anchors for arthroscopic Bankart repairs with a strong biomechanical profile and promising clinical short-term results $[1,2,5]$. The benefits of knotless, all-suture anchors include the small size, allowing for more points of fixation per unit area, improved access due to the ability to insert through curved drill guides, artifactreduced postoperative imaging, bone stock preservation, less risk of erosion of the humeral head or soft tissue such as the rotator cuff, and time efficiency due to the knotless construct [1-5].

\section{Operative technique}

The senior author's preferred technique utilizes the beach chair position, but the lateral decubitus position is also routinely used. Diagnostic arthroscopy is performed through a standard posterior viewing portal to identify the pathology

\author{
Anna-K. Tross ${ }^{1,2} \cdot$ Philip-C. Nolte ${ }^{1,3} \cdot$ Joseph J. Ruzbarsky' $\cdot$ Bryant P. Elrick' \\ Adam M. Johannsen ${ }^{1} \cdot$ Peter J. Millett ${ }^{4}$ \\ 'Steadman Philippon Research Institute, Vail, USA \\ ${ }^{2}$ Clinic for Orthopedics and Trauma Surgery, Heidelberg University Hospital, Heidelberg, Germany \\ ${ }^{3}$ Clinic for Trauma and Orthopaedic Surgery, BG Trauma Center Ludwigshafen at the University of \\ Heidelberg, Ludwigshafen, Germany \\ ${ }^{4}$ Steadman Philippon Research Institute, The Steadman Clinic, Vail, USA
}

\title{
Arthroscopic Bankart repair with knotless all-suture anchors
}

at the anteroinferior aspect of the glenoid and to address any potential concomitant lesions.

Under direct visualization while utilizing a $30^{\circ}$ arthroscope, an anterosuperior portal is created and placed high into the rotator interval. The senior author creates a third portal, the anteroinferior portal, which is established just above the upper border of the subscapularis tendon providing adequate trajectory for later anchor placement and passage of sutures around the labrum and capsule. An 8.25$\mathrm{mm}$ cannula is typically used in this location in order to accommodate $25^{\circ}$ and $45^{\circ}$ curved suture-passing instruments (- Fig. 1).

The capsulolabral complex is mobilized with the help of an arthroscopic periosteal elevator. A $70^{\circ}$ scope can also be used at this point to help with visualization. Adequate mobilization is achieved when the muscle fibers of the subscapularis are visualized and the resting position of the elevated capsulolabral complex is at the level of the glenoid cartilage. The capsulolabral sleeve can be shifted to assess the desired tension. The glenoid neck is then debrided to a bleeding bony bed with an oscillating arthroscopic burr to promote healing. The cortex is preserved, however, so as not to weaken anchor fixation or increase the risk of postsurgical fracture/erosion. The first anchor is strategically placed at the 5:30-o'clock position in order to shift the inferior capsulolabral complex and to tighten the axillary pouch. A curved drill guide is inserted through the an- teroinferior portal to pilot the drill hole at the anteroinferior glenoid rim. The first knotless, all-suture anchor $(1.8 \mathrm{~mm}$ FiberTak, Arthrex, Naples, FL, USA) is placed through the drill guide into the previously drilled hole, and is then manually provisionally seated, followed by mallet impaction for full seating into the bone. Tension is applied to the anchor sutures in order to allow the anchor to sit beneath the cortex. Once the anchor stability is checked, suture management can be initiated.

There are three suture limbs per anchor: one repair suture (white-blue) and two limbs of the shuttle suture (whiteblack). The repair suture is separated from the shuttling suture by retrieving it through the anterosuperior portal using an atraumatic arthroscopic grasping instrument (• Fig. 2). The senior author prefers to use a $25^{\circ}$ angulated sutureshuttling device that is passed through the anteroinferior $8.25-\mathrm{mm}$ cannula and then through the desired capsulolabral tissue in order to facilitate a capsular shift from lateral to medial and from inferior to superior. The nitinol wire of the shuttling device is then retrieved through the anterosuperior portal. The repair suture is placed into the loop of the nitinol wire and is shuttled back through the anteroinferior portal, completing the repair suture passage.

The repair suture is now threaded through the loop of the shuttle suture. The non-looped shuttle suture is then pulled gently until the repair suture passes back through the anteroinferior 


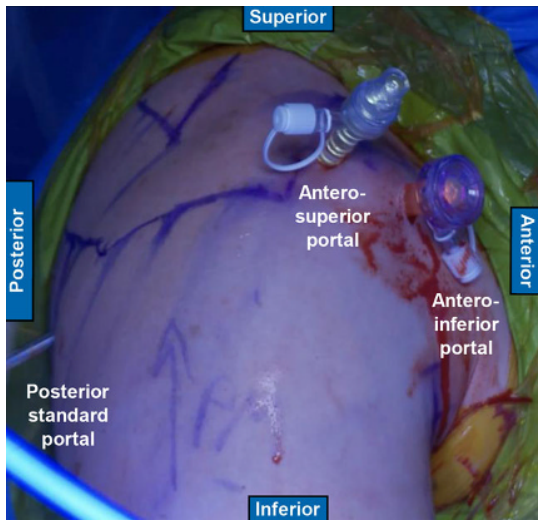

Fig. $1 \Delta$ Right shoulder with arthroscopic portals: standard posterior viewing portal, anterosuperior portal, and anteroinferior portal



Fig. $2 \Delta$ Arthroscopic image of a right shoulder. The repair suture (white-blue) is separated from the shuttling suture (white-black) by retrieving it through the anterosuperior portal using an atraumatic arthroscopic grasping instrument

risk of anchor arthropathy, and reduced bone reactions (e.g., osteolysis) around the implant when compared with solid biocomposite anchors [4]. Concerns about limited failure load, anchor displacement, and anchor pull-out were recently addressed by Lacheta et al. [2], who demonstrated high loads to failure for both knotted and knotless all-suture anchor repairs, with superior loop security of knotless repairs. Furthermore, Ntalos et al. [3] postulated that even in the case of pull-out, all-suture anchors may be advantageous over solid suture anchors as there is less potential for articular cartilage damage. All-suture anchors also remain permanently in the bone and soft tissue possibly acting to reinforce the damaged tissues. Clinical outcome studies following all-suture anchor Bankart repairs are still scarce and mostly limited to short-term followup; however, early results are promising. Investigating 20 patients following arthroscopic Bankart repair with allsuture anchors at a minimum follow-up of 1 year, Willemot et al. [5] did not observe recurrent instability. Likewise, Gül et al. [1] demonstrated good-toexcellent clinical outcome scores (Rowe and Constant score) in over $90 \%$ of 62 patients examined at a minimum followup of 24 months.

\section{Practical conclusion}

- All-suture anchors for Bankart repair are a valuable alternative to solid suture anchors, with benefits comprising the small size, the ability to be inserted through curved drill guides allowing for more versatile access to the glenoid rim, artifact-reduced postoperative imaging, and bone stock preservation.

- Knotless all-suture anchor constructs potentially reduce operative time as knot-tying is omitted, while decreasing the risk of cartilage abrasion and anchor arthropathy due to knot stacks or proud anchors.

- Early clinical results are promising; however, longer-term follow-up studies, especially for knotless constructs, are needed.

\section{Corresponding address}



Peter J. Millett, MD, MSc

Steadman Philippon Research Institute, The Steadman Clinic 181 W Meadow Dr, Ste 400, 81657 Vail, CO, USA drmillett@ thesteadmanclinic.com

\section{Compliance with ethical guidelines}

Conflict of interest. The position of P.-C. Nolte at the Steadman Philippon Research Institute was supported by Arthrex. P.J. Millett is a consultant for and receives royalties from Arthrex, Medbridge, and Springer; owns stock in VuMedi; receives support from the Steadman Philippon Research Institute and Vail Valley Medical Center; and has corporate sponsorship from the Steadman Philippon Research Institute, Smith \& Nephew, Arthrex, Siemens, and Össur. A.-K. Tross, J. Ruzbarsky, B.P. Elrick and A.M. Johannsen declare that they have no competing interests.

For this article no studies with human participants or animals were performed by any of the authors. All studies performed were in accordance with the ethical standards indicated in each case.

Open Access. This article is licensed under a Creative Commons Attribution 4.0 International License, which permits use, sharing, adaptation, distribution and reproduction in any medium or format, as long as you give appropriate credit to the original author(s) and the source, provide a link to the Creative Commons licence, and indicate if changes were made. The images or other third party material in this article are included in the article's Creative Commons licence, unless indicated otherwise in a credit line to the material. If 
material is not included in the article's Creative Commons licence and your intended use is not permitted by statutory regulation or exceeds the permitted use you will need to obtain permission directly from the copyright holder. To view a copy of this licence, visit http://creativecommons.org/licenses/by/4.0/.

\section{References}

1. Gül O, Okutan AE, Ayas MS (2019) Arthroscopic glenoid labral lesion repair using all-suture anchor for traumatic anterior shoulder instability: short-term results. J Shoulder Elbow Surg 28(10):1991-1997. https://doi.org/10.1016/j.jse. 2019.03.003

2. Lacheta L, Brady A, Rosenberg SI et al (2020) Biomechanical evaluation of knotless and knotted all-suture anchor repair constructs in four Bankart repair configurations. Arthroscopy 36(6):1523-1532. https://doi.org/10.1016/j. arthro.2020.01.046

3. Ntalos D, Huber G, Sellenschloh K et al (2020) Allsuture anchor pullout results in decreased bone damage and depends on cortical thickness. Knee Surg Sports Traumatol Arthrosc. https://doi.org/ 10.1007/s00167-020-06004-6

4. Stewart CMB, Raja H, Torrance E et al (2020) In vivo randomized controlled study of the bone response of all-suture anchors and biocomposite anchors. Orthop J Sports Med 8:2325967120914965

5. Willemot L, Elfadalli R, Jaspars KC et al (2016) Radiological and clinical outcome of arthroscopic labral repair with all-suture anchors. Acta Orthop Belg 82:174-178 